\title{
Physical characterization of the organic nonlinear optical crystal - 3-methoxy 4-hydroxy benzaldehyde
}

\author{
V VENKATARAMANAN, SANJAY UCHIL and H L BHAT \\ Department of Physics, Indian Institute of Science, Bangalore 560012, India
}

\begin{abstract}
We highlight our recent experimental work on an efficient molecular nonlinear optical crystal, 3-methoxy 4-hydroxy benzaldehyde (MHBA). Optical quality single crystals of MHBA were grown from mixtures of solvents and from melt. The overall absorption and transparency window were improved by growing them in a mixture of chloroform and acetone. The grown crystals were characterized for their optical transmission, mechanical hardness and laser damage. We have observed a strong correlation between mechanical properties and laser induced damage.
\end{abstract}

\section{Introduction}

High storage density optical devices require laser sources at short wavelengths, typically around the blue region. Direct compact sources are currently not available at these wavelengths. A viable alternative is to obtain them by intra-cavity frequency doubling of GaAs-AlGaAs lasers. The requirements for such crystals are very stringent; they should have high nonlinearity, high angular acceptance and high damage threshold. Till today the only inorganic crystal in which intra-cavity frequency doubling of $\mathrm{GaAs}$ lasers was demonstrated is $\mathrm{KNbO}_{3}$. However, this requires thermal stabilization of the cavity as the thermo-optic coefficients of $\mathrm{KNbO}_{3}$ are high, which is a serious handicap in device fabrication. Hence alternative materials are currently being explored, and 3-methoxy 4-hydroxy benzaldehyde promises to be a good candidate. MHBA is an efficient molecular nonlinear optical (NLO) material. It has relatively weak donor and acceptor $(-\mathrm{CHO}$ and $-\mathrm{OH})$ at para positions in a benzene ring and $-\mathrm{OCH}_{3}$ group attached at the meta position, ensuring an acentric crystal structure. Its SHG powder efficiency is as high as 30 times that of urea and it has transparency in the blue region. It has a low threshold for SHG and low angular sensitivity. Also, crystals of MHBA can be grown from solutions at low temperature. A combination of these desirable properties makes MHBA an excellent material for investigation as a frequency doubler for GaAs lasers. In this paper we discuss some of our recent experiments on this important organic NLO crystal.

\section{Experimental and results}

\subsection{Crystal growth}

Single crystal of MHBA were grown from various solvents and solvent mixtures using an indigenous growth unit fabricated in our laboratory (Dhanaraj et al 1991). It was observed during our growth experiments that MHBA, owing to its highly polar nature, 
grows as needles and rods from various solvents. Hence single crystals were grown from various solvent mixtures. The results of our extensive investigations on the effect of solvent and solvent mixtures on the crystal habit and optical quality of MHBA have already been published in one of our earlier papers (Venkataramanan et al 1994a).

Since it was very difficult to grow large single crystals of MHBA from solvents or solvent mixtures, we considered it worthwhile to attempt other methods of growth as well. As a first step towards melt growth, we studied the behaviour of MHBA at the melting point. Our DSC (figure 1) and TGA experiments showed that the compound does not sublime and is quite stable up to its melting point. These results indicated that MHBA could be grown from melt. Subsequently, large single crystals of MHBA of size $30 \times 20 \times 2 \mathrm{~mm}^{3}$ were grown from melt (figure 2). In general, the melt grown crystals were yellowish in colour indicating increased optical absorption. However, unlike the solution grown crystals, these crystals did not suffer from inclusions and their preferential incorporation into certain regions (Venkataramanan et al 1994a).

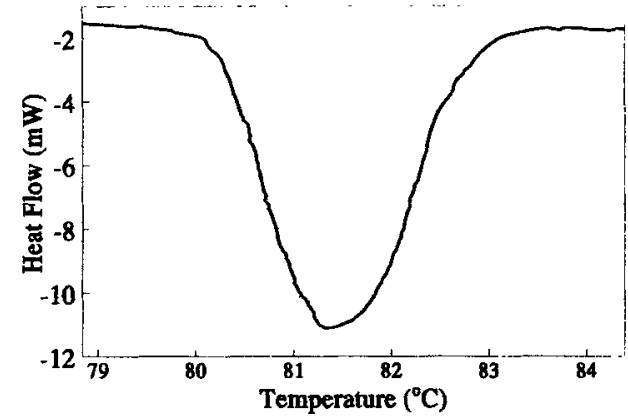

Figure 1. DSC curve of MHBA.

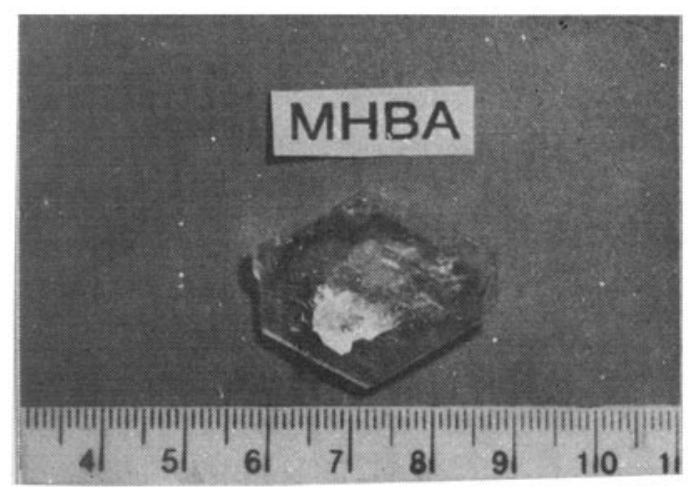

Figure 2. Pholograph of a typical melt grown crystal. 


\subsection{Optical transmission}

Molecular NLO crystals usually have high absorption in the visible wavelength and narrow transparency window. This is one of the disadvantages of these materials which limits their utility. The high excited-to-ground state dipole moment that increases the nonlinearity also increases the absorption. MHBA, however, has only weak donor and acceptor and hence can be expected to have lower absorption than the other organic crystals.

Figure 3 shows the optical transmission of MHBA in the region $200-2000 \mathrm{~nm}$. MHBA was found to have low absorption at the Nd: YAG fundamental wavelength, which contributes to its resistance to laser induced damage. Also, there was very little absorption at the doubled wavelength $(532 \mathrm{~nm})$, which can improve the second harmonic throughput. It was also found to have an extended transmission range (down to $360 \mathrm{~nm}$ ). This, coupled with the higb nonlinearity makes MHBA very attractive for diode laser frequency doubling applications as well as those with tunable Ti-sapphire lasers.

On visual examination, the-crystals grown from chloroform-acetone mixture were found to be less coloured than those from acetic acid-water mixture. Optical absorption studies were hence carried out on samples grown from different solutions, which yielded crystals large enough for characterization. Particularly, crystals grown from acetic acid-water and chloroform-acetone were chosen for comparison. The inset in figure 3 shows the low wavelength cut-off of the crystals grown from acetic acid $(370 \mathrm{~nm})$ and those from chloroform mixtures $(365 \mathrm{~nm})$. It was observed that in samples of identical thickness, the crystals grown from chloroform-acetone mixture had approximately $10 \%$ less absorption than those grown from the other mixture. It should be emphasized here that though the mixture of acetic acid-water gave larger crystals (Zhang et al 1992), crystals grown from chloroform-acetone mixture, with higher transparency range and low overall absorption are more suitable for optical experiments. As regards to the melt grown crystals, there was an overall

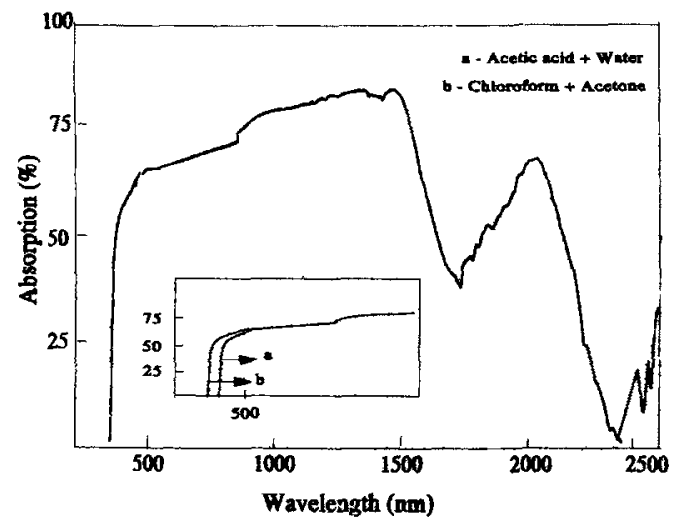

Figure 3. Optical transmission of MHBA cTystals grown from chloroform-acetone mixtures. The inset shows the increase in transparency when compared to the crystals grown from acetic acid-water mixture. 
increase in optical absorption as compared to those grown from solutions. Further optimization of the growth conditions is required to improve the optical transmission in the melt grown crystals.

\subsection{Defects characterization}

The grown MHBA crystals were characterized for defect content using X-ray topography employing the synchrotron radiation source at Daresbury Laboratory, UK. The surface damage from cleavage was removed by wet polishing the crystal. The crystal plate of $2 \mathrm{~mm}$ thickness was set to 400 reflection for $0.72 \AA$ radiation of the white radiation from a synchrotron source. Figure 4 shows a typical synchrotron topograph of a MHBA crystal grown from chloroform-acetone mixture. In general, the dislocation densities were very high, as the crystals were self-nucleated. Consequently, the individual dislocations could not be resolved. This topograph reflects the typical characteristics of organic crystals, showing high contrast variation and poor resolution (Halfpenny et al 1993).

Figure 5 is the synchrotron topograph of a MHBA crystal grown from melt. As can be seen from the figure the melt grown crystals were highly strained. As a result of the large strain, some regions of topograph are seen in out of diffraction conditions, which results in an incomplete imaging of the crystal. An improvement in the overall quality should be possible with improved growth conditions. Work is currently under progress to optimize the growth conditions.

\subsection{Laser induced damage}

Previous report on laser damage indiciled a threshold value in excess of $1 \mathrm{GW} / \mathrm{cm}^{2}$ for MHBA for a $25 \mathrm{~ns}$ pulse (Yakovlev and Poezzhalov 1991). However, they were unable to accurately measure the damage threshold of MHBA. Hence, a measurement of laser damage resistance was made on the MHBA crystals grown from solutions.

For laser damage experiments a picosecond Continuum YG601 Nd:YAG laser

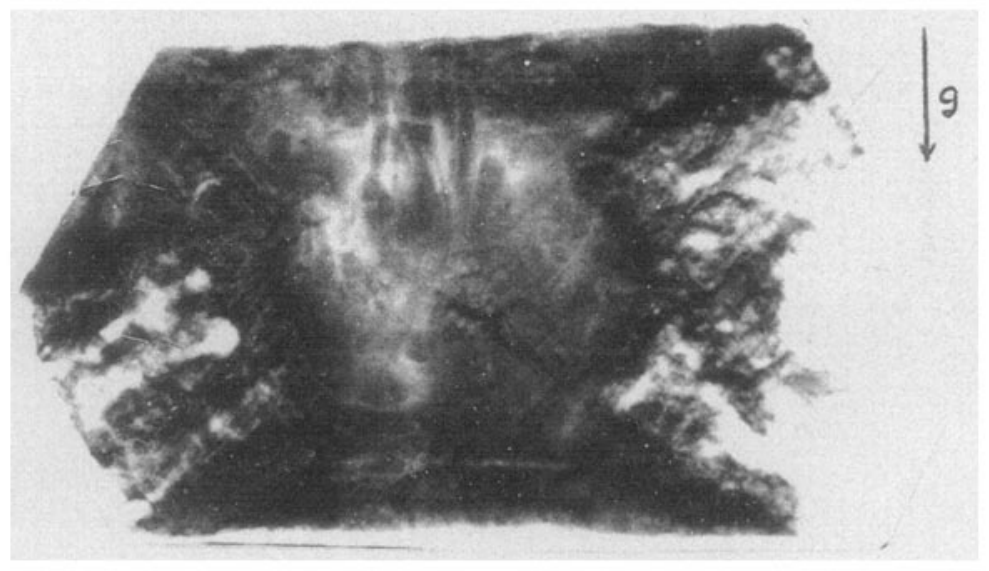

Figure 4. Synchrotion topograph of MHBA crystal grown from solution, $\lambda=0.72 \AA, 400$ reflection, 001 plate. 
was used. The experimental set-up for evaluating laser induced damage is shown in figure 6. The combination of polarizer and the attenuator serves to cut the input intensity to the required level. The beam splitter transmits $15 \%$ of the power to the pyroelectric probe (Laser Precision Corporation, RjP-7735). The power meter was triggered by synchronous pulses from the Q-switch of the Nd: YAG laser. The crystals were kept slightly away from the focal spot of the beam. A low power He-Ne laser beam was also simultaneously focussed onto the crystal. When damage occurs, this $\mathrm{He}-\mathrm{Ne}$ beam gets scattered which could easily be detected even by naked eye. The transmitted He-Ne beam from the crystal was collected on a screen for further facilitating the detection of damage occurrence.

MHBA samples were prepared by cleaving the crystals in the $(001)$ plane. As

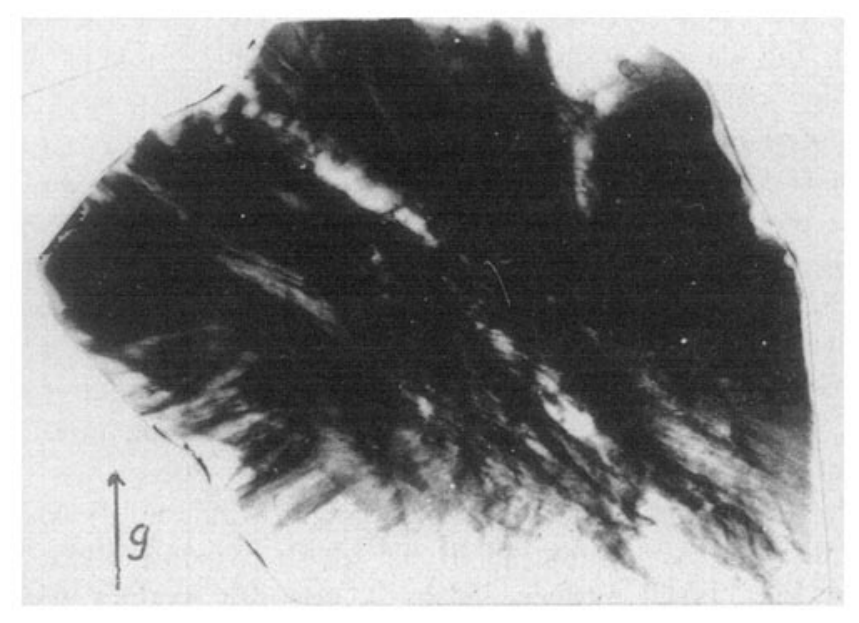

Figure 5. Synchrotron topograph of MHBA crystal grown from melt. $\lambda=0.72 \AA, 400$ reflection, 001 plate.

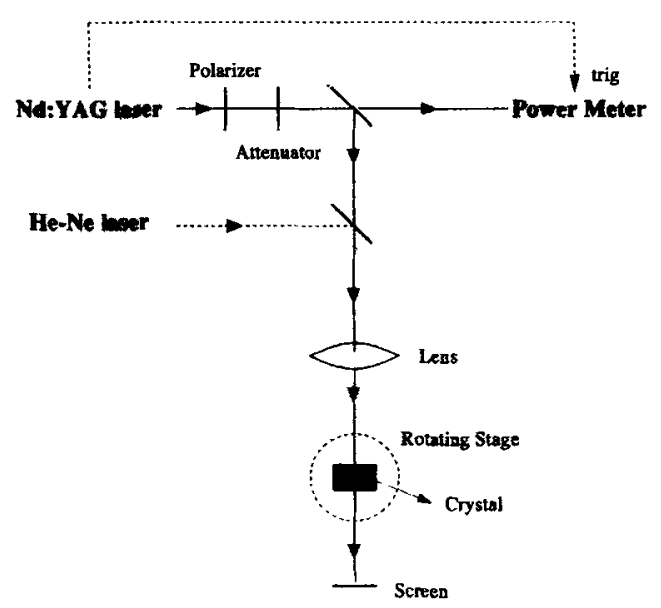

Figure 6. Experimental arrangement for laser damage measurements. 
the cleavage was facile and smooth without any steps, no polishing was required. No special cleaning was done to the surface. The crystals selected for the experiments were of the best quality among those grown. Since MHBA crystals always grow with inclusions in negative $b$ axis, exposures were restricted to the other end. Pre-exposure examination in selecting the test site included visual examination for inclusions and looking for light scattering from the He-Ne laser used as the probe in the actual experimental arrangement.

During single shot experiments to measure 1-on-1 $\left(P_{1}\right)$ damage resistance values, the laser was operated by a remote control. The occurrence of damage (or otherwise) was monitored on the screen and irrespective of whether the damage had occurred or not, the samples were moved to a new site. The distance between the two sites was kept at least 5 times the spot size on the crystal surface.

During multiple shot experiments $n$-on-1 $\left(P_{n}\right)$, the laser beam at sub-threshold value was made to fall on same site of the crystal and the onset of damage (the scattering of He-Ne beam) was monitored on the screen. The laser power was continuously monitored and averaged for the entire duration of the experiment. If the damage had not occurred even after 2000 pulses, the crystal was moved so that the beam fell at a new site and the experiment was repeated with slightly higher power. This procedure was repeated till the test site was damaged with 2000 pulses. The value of $P_{n}$ was averaged over a large number of observations.

The laser parameters and the results obtained are given in table 1 . It can be seen that the damage threshold values obtained during the present investigations are less than that reported earlier, considering the fact that the present study is with picosecond pulses. In their experiments, Yakovlev and Poezzhalov (1991), had kept the crystals in an evacuated chamber with quartz windows. It was not clear if they had accounted for the absorption in the quartz window while calculating the total power at the crystal surface. Also, it was not explicit whether their experimental values corresponded to the bulk damage measurements, in which case it can be an order of magnitude higher than the surface threshold. During the present investigation, the crystals were directly exposed to focused radiation without any enclosures and the values reported here are for surface damage threshold.

Table 1. Laser damage in MHBA - Test parameters and results.

\begin{tabular}{|c|c|}
\hline \multicolumn{2}{|c|}{ Test parameters } \\
\hline Wavelength & $532 \mathrm{~nm}$ \\
\hline Repetition rate & $5 \mathrm{~Hz}$ \\
\hline Pulse width & $32 \mathrm{psec}$ \\
\hline Beam profile & TEMoo \\
\hline Beam diameter & $3.6 \mathrm{~mm}$ (at $1 / \mathrm{e}^{2}$ ) \\
\hline Specimen orientation & $(001)$ \\
\hline Polarization & perp. to $b$ \\
\hline \multicolumn{2}{|c|}{ Results } \\
\hline Single shot $\left(P_{1}\right)$ & $7 \mathrm{GW} / \mathrm{cm}^{2}$ \\
\hline Multiple shot $\left(P_{n}\right)$ & $0.8 \mathrm{GW} / \mathrm{cm}^{2}$ \\
\hline
\end{tabular}




\subsection{Mechanical hardness}

It was speculated previously that a knowledge of the mechanical properties of NLO crystals is essential in understanding the origin of laser induced damage in them (Yokotani et al 1989). With this in view, we carried out mechanical hardness studies on these crystals. The anisotropy of hardness of MHBA was evaluated on the cleavage (001) plane by Knoop indentation. A Leitz Miniload tester fitted with a Knoop indenter was used. A constant load of $15 \mathrm{~g}$ was applied for all indentations. The descending time was kept constant at $20 \mathrm{sec}$ and the dwell time $40 \mathrm{sec}$. Starting from the $a$ axis, indentations were made for incremental rotations of $10^{\circ}$ in the entire (001) plane and the KHN were averaged over a large number of values. Figure 7 shows the variation of mechanical hardness as a function of angle. As can be inferred from the graph, there is a marked anisotropy in hardness of MHBA. Hardness along $b$ axis is higher than that along the $a$ axis. In general, the hardness of MHBA is quite lower when compared to those of inorganic NLO crystals. The lower hardness value can be attributed to the presence of relatively weak bonds in the MHBA molecule. The information on hardness anisotropy was subsequently used in understanding the nature of laser induced damage in the crystal, as outlined below.

The morphology of the damage pattern can reveal the nature and possible origin of damage in the crystals. The damage patterns often reflect the symmetries of the crystal surface. Earlier workers have predicted the origin of laser damage through a careful examination of these patterns, which included dielectric breakdown, thermal decomposition, etc (Ellenberger et al 1992; Bolt and van der Mooren 1993). However, no specific correlation between the mechanical hardness anisotropy and the nature of laser damage has been made so far. To observe the possible correlation, we examined the morphologies of laser induced damages in MHBA and compared them with mechanical hardness anisotropy. Post-exposure examination of the damaged surfaces revealed highly anisotropic damage profiles, the orientation of which were identified from the morphology of the samples. The elliptical laser damage has its major and minor axes along the crystallographic $b$ and $a$ axes (figure 8a). The polar plot of hardness anisotropy (figure $8 b$ ) was compared with the damage morphology.

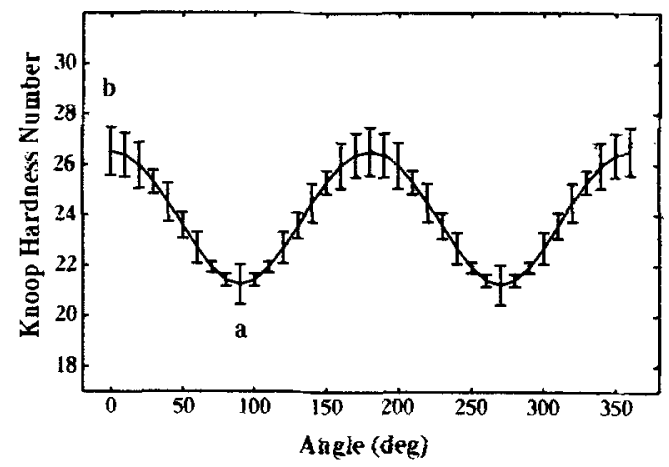

Figure 7. Mechanical hardness anisotropy of MHBA in the (001) plane. 

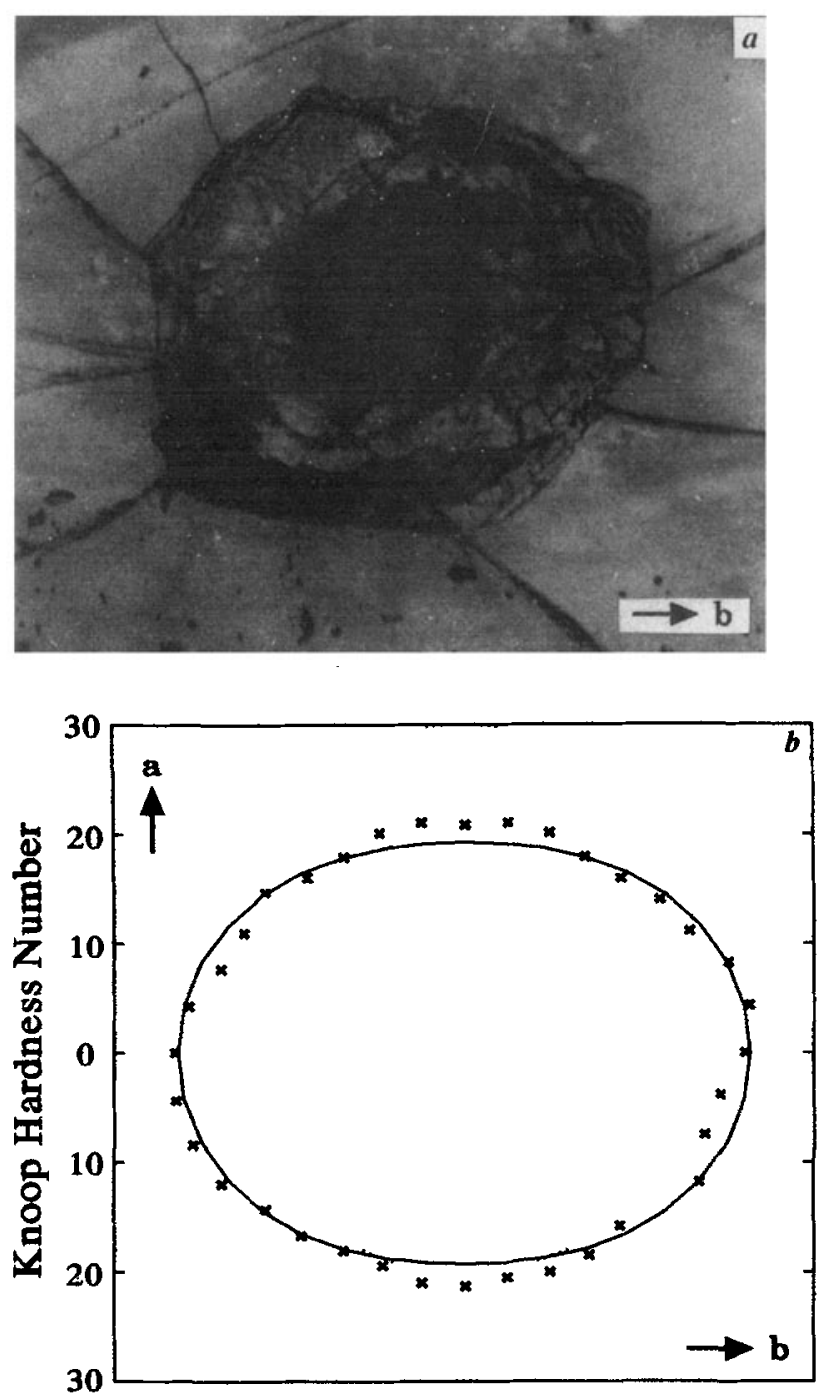

Figure 8. a. Optical micrograph of laser induced damage in 001 plane of MHBA $(\times 60)$ and b. polar plot of hardness anisotropy of MHBA.

It was observed that the laser damage was severe along the mechanically harder $b$ axis. This is in consistence with our reports on other NLO crystals, viz. L-arginine phosphate monohydrate (LAP) (Venkataramanan et al 1994b) zinc tris(thiourea) sulfate (ZTS) and bis(thiourea) cadmium chloride (BTCC) (Venkataramanan et al 1994c). However, unlike the other crystals studied by us, MHBA has a sharp damage threshold value. Just above the threshold there were severe fragmentation and fracture, leading to the physical removal of material from the surface. We attribute this to the presence of a facile cleavage plane in MHBA. It should, however, be reiterated that the mechanism of laser induced damage is highly complicated and case-specific. Detailed studies are warranted on different crystals under identical experimental conditions to develop a comprehensive model. 


\section{Conclusions}

Single crystals of MHBA were grown from various solvents, solvent mixtures and melt. Spectrophotometric studies on single crystals revealed that crystals grown in a mixture of chloroform and acetone have lesser absorption than those grown from other solvent mixtures. Also crystals from chloroform-acetone have increased transparency window and are thus better suited for optical applications. Detailed thermal analysis on the compound revealed that MHBA does not have any high temperature phase transition and does not sublime appreciably till its melting point. Mechanical hardness assessment on MHBA indicates that the crystals are softer than semiorganics. Laser damage threshold experiments revealed a high resistance to laser damage and the damage morphologies were found to have strong correlation with mechanical hardness anisotropy.

\section{Acknowledgements}

The authors thank Prof. J N Sherwood for fruitful discussions. The topographic work was carried out at Daresbury laboratory, UK under the financial support from the British Council. The authors are grateful to Prof. B M Sivaram, IIT, Madras for extending his laser facility and $\mathrm{Mr}$ Vetrivel for the help in experiments. One of the authors (VV) acknowledges UGC for a Senior Research Fellowship.

\section{References}

Boit R J and van der Mooren 1993 Opt. Commun. 100399

Dhanaraj G, Shripathi T and Bhat H L 1991 J. Cryst. Growth 113456

Ellenberger U, Weber R, Balmer J E, Zysset B, Ellgehausen D and Mizell G J 1992 Appl. Opt. 31 7563

Halfpenny P J, Morrison H, Ristic R I, Shephered E E A, Sherwood J N, Simpson G S and Yoon C S 1993 Proc. R. Soc. London A440 683

Venkataramanan V, Sanjay Uchil, Subramanian C K and Bhat H L 1994a, Proc. of the conf. on emerging optoelectron. technol, pp 71-74

Venkataramanan V, Dhanaraj G, Prasad B R, Subramanian C K and Bhat H L 1994b Ferroelectrics 15513

Venkataramanan V, Subramanian C K and Bhat H L 1994c Appl. Phys. Lett. (Communicated)

Yakovlev Yu O and Poezzhalov V M 1991 Sov. J. Quantum. Electron. 21483

Yokotani A, Sasaki T, Yoshida K and Nakai S 1989 Appl. Phy.s. Lett. 552692

Zhang N, Yuan D, Shao Z, Dou S, Jiang M and Xu D 1992 J. Cryst. Growth 123255 